\title{
A NOTE ON POISSON LIE ALGEBROIDS
}

\section{LIVIU POPESCU}

Communicated by Izu Vaisman

\begin{abstract}
In this paper we study some properties of a Lie algebroid and its prolongation over the vector bundle projection of the dual bundle. We generalize some results on Poisson manifolds to the level of a Lie algebroid. The notions of canonical Poisson bivector and horizontal lift are studied and their compatibility conditions are pointed out.
\end{abstract}

\section{Introduction}

The Lie algebroid [10] is a generalization of both concepts of Lie algebra and integrable distribution, being a vector bundle $(E, \pi, M)$ with a Lie bracket on his space of sections with properties very similar to those of a tangent bundle. The Poisson manifolds are the smooth manifolds equipped with a Poisson bracket on their ring of functions. I have to remark that the cotangent bundle of a Poisson manifold has the natural structure of a Lie algebroid [13]. In the last years diverse aspects of these subjects have been studied in a lot of papers (see for instance [13], [14], [12], [1] and [7]). In the present paper we study some geometrical structures on the prolongation of a Lie algebroid to its dual bundle and investigate some aspects of the Lie algebroid geometry endowed with a Poisson structure. In this way we generalize some results on Poisson manifolds.

The paper is organized as follows. In the Section 2 we recall the Cartan calculus and the Schouten-Nijenhuis bracket at the level of a Lie algebroid and present the Poisson structures on the Lie algebroid. The Section 3 deals with the prolongation of a Lie algebroid [5], [8] to its dual bundle and continue the investigation starting in [6]. We study the properties of the canonical Poisson bivector and introduce the notion of horizontal lift. Finally, the compatibility conditions of these bivectors are given. We remark that in the particular case of the standard Lie algebroid $(E=T M, \sigma=\mathrm{Id})$ some results of Mitric [12] are obtained. 


\section{Preliminaries on Lie Algebroids}

Let $M$ be a differentiable, $n$-dimensional manifold and $\left(T M, \pi_{M}, M\right)$ its tangent bundle. A Lie algebroid over the manifold $M$ is the triple $(E,[\cdot, \cdot], \sigma)$ where $\pi$ : $E \rightarrow M$ is a vector bundle of rank $m$ over $M$, whose $C^{\infty}(M)$-module of sections $\Gamma(E)$ is equipped with a Lie algebra structure $[\cdot, \cdot]$ and $\sigma: E \rightarrow T M$ is a vector bundle map (called the anchor) which induces a Lie algebra homomorphism (also denoted $\sigma$ ) from $\Gamma(E)$ to $\chi(M)$, satisfying the Leibnitz rule

$$
\left[s_{1}, f s_{2}\right]=f\left[s_{1}, s_{2}\right]+\left(\sigma\left(s_{1}\right) f\right) s_{2}
$$

for every $f \in C^{\infty}(M)$ and $s_{1}, s_{2} \in \Gamma(E)$. Therefore, we have

$$
\left[\sigma\left(s_{1}\right), \sigma\left(s_{2}\right)\right]=\sigma\left[s_{1}, s_{2}\right], \quad\left[s_{1},\left[s_{2}, s_{3}\right]\right]+\left[s_{2},\left[s_{3}, s_{1}\right]\right]+\left[s_{3},\left[s_{1}, s_{2}\right]\right]=0 .
$$

If $\omega \in \bigwedge^{k}\left(E^{*}\right)$ then the exterior derivative $\mathrm{d}^{E} \omega \in \bigwedge^{k+1}\left(E^{*}\right)$ is given by the formula

$$
\begin{aligned}
\mathrm{d}^{E} \omega\left(s_{1}, \ldots, s_{k+1}\right)= & \sum_{i=1}^{k+1}(-1)^{i+1} \sigma\left(s_{i}\right) \omega\left(s_{1}, \ldots, \hat{s}_{i}, \ldots, s_{k+1}\right) \\
& +\sum_{1 \leq i<j \leq k+1}(-1)^{i+j} \omega\left(\left[s_{i}, s_{j}\right], s_{1}, \ldots, \hat{s}_{i}, \ldots, \hat{s}_{j}, \ldots s_{k+1}\right) .
\end{aligned}
$$

where $s_{i} \in \Gamma(E), i=\overline{1, k+1}$, and it follows that $\left(\mathrm{d}^{E}\right)^{2}=0$. Also, for $\xi \in \Gamma(E)$ one can define the Lie derivative with respect to $\xi$ by

$$
\mathcal{L}_{\xi}=i_{\xi} \circ \mathrm{d}^{E}+\mathrm{d}^{E} \circ i_{\xi}
$$

where $i_{\xi}$ is the contraction with $\xi$.

If we take the local coordinates $\left(x^{i}\right)$ on an open $U \subset M$, a local basis $\left\{s_{\alpha}\right\}$ of sections of the bundle $\pi^{-1}(U) \rightarrow U$ generates the local coordinates $\left(x^{i}, y^{\alpha}\right)$ on $E$. The local functions $\sigma_{\alpha}^{i}(x), L_{\alpha \beta}^{\gamma}(x)$ on $M$ defined by

$$
\sigma\left(s_{\alpha}\right)=\sigma_{\alpha}^{i} \frac{\partial}{\partial x^{i}}, \quad\left[s_{\alpha}, s_{\beta}\right]=L_{\alpha \beta}^{\gamma} s_{\gamma}, \quad i=1, \ldots, n, \quad \alpha, \beta, \gamma=1, \ldots, m
$$

are called the structure functions of the Lie algebroid and satisfy the so called structure equations on the Lie algebroid

$$
\sigma_{\alpha}^{j} \frac{\partial \sigma_{\beta}^{i}}{\partial x^{j}}-\sigma_{\beta}^{j} \frac{\partial \sigma_{\alpha}^{i}}{\partial x^{j}}=\sigma_{\gamma}^{i} L_{\alpha \beta}^{\gamma}, \quad \sum_{(\alpha, \beta, \gamma)}\left(\sigma_{\alpha}^{i} \frac{\partial L_{\beta \gamma}^{\delta}}{\partial x^{i}}+L_{\alpha \eta}^{\delta} L_{\beta \gamma}^{\eta}\right)=0 .
$$


Locally, if $f \in C^{\infty}(M)$ then $\mathrm{d}^{E} f=\frac{\partial f}{\partial x^{i}} \sigma_{\alpha}^{i} s^{\alpha}$, where $\left\{s^{\alpha}\right\}$ is the dual basis of $\left\{s_{\alpha}\right\}$ and, if $\theta \in \Gamma\left(E^{*}\right), \theta=\theta_{\alpha} s^{\alpha}$ then

$$
\mathrm{d}^{E} \theta=\left(\sigma_{\alpha}^{i} \frac{\partial \theta_{\beta}}{\partial x^{i}}-\frac{1}{2} \theta_{\gamma} L_{\alpha \beta}^{\gamma}\right) s^{\alpha} \wedge s^{\beta} .
$$

Particularly, we get

$$
\mathrm{d}^{E} x^{i}=\sigma_{\alpha}^{i} s^{\alpha}, \quad \mathrm{d}^{E} s^{\alpha}=-\frac{1}{2} L_{\beta \gamma}^{\alpha} s^{\beta} \wedge s^{\gamma} .
$$

The Schouten-Nijenhuis bracket is given by [13]

$$
\begin{gathered}
{\left[X_{1} \wedge \ldots \wedge X_{p}, Y_{1} \wedge \ldots \wedge Y_{q}\right]} \\
=(-1)^{p+1} \sum_{i=1}^{p} \sum_{j=1}^{q}(-1)^{i+j}\left[X_{i}, Y_{j}\right] \wedge X_{1} \wedge \ldots \wedge \hat{X_{i}} \wedge \ldots \\
\wedge X_{p} \wedge Y_{1} \wedge \ldots \wedge Y_{j} \wedge \ldots \wedge Y_{q}
\end{gathered}
$$

where $X_{i}, Y_{j} \in \Gamma(E)$ and a hat means the absence of a factor.

\subsection{Lie Algebroids with Poisson Structure}

Let us consider the bivector on $E$ (i.e., contravariant, skew-symmetric, 2-section) $W \in \Gamma\left(\wedge^{2} E\right)$ given by

$$
W=\frac{1}{2} w^{\alpha \beta}(x) s_{\alpha} \wedge s_{\beta} .
$$

Definition 1. The bivector $W$ is a Poisson bivector on $E$ if and only if we have the relation $[W, W]=0$, where [,] is Schouten-Nijenhuis bracket.

Proposition 2. The relation $[W, W]=0$ implies locally that

$$
\sum_{(\alpha, \varepsilon, \delta)}\left(w^{\alpha \beta} \sigma_{\beta}^{i} \frac{\partial w^{\varepsilon \delta}}{\partial x^{i}}+w^{\alpha \beta} w^{\gamma \delta} L_{\beta \gamma}^{\varepsilon}\right)=0 .
$$

If $W$ is a Poisson bivector then the pair $(E, W)$ is called a Lie algebroid with Poisson structure. The Poisson bracket on $E$ is given by

$$
\left\{f_{1}, f_{2}\right\}=W\left(\mathrm{~d}^{E} f_{1}, \mathrm{~d}^{E} f_{2}\right), \quad f_{1}, f_{2} \in C^{\infty}(E) .
$$

We have the bundle map $\pi^{\#}: E^{*} \rightarrow E$ defined by

$$
\pi^{\#} \rho=i_{\rho} W, \quad \rho \in \Gamma\left(E^{*}\right) .
$$


Let us consider the bracket

$$
[\rho, \theta]_{\pi}=\mathcal{L}_{\pi \# \rho} \theta-\mathcal{L}_{\pi \# \theta} \rho-\mathrm{d}^{E}(W(\rho, \theta))
$$

where $\mathcal{L}$ is the Lie derivative and $\rho, \theta \in \Gamma\left(E^{*}\right)$. With respect to this bracket and the usual Lie bracket on vector fields, the map $\widetilde{\sigma}: E^{*} \rightarrow T M$ given by

$$
\widetilde{\sigma}=\sigma \circ \pi^{\#}
$$

is a Lie algebra homomorphism

$$
\widetilde{\sigma}[\rho, \theta]_{\pi}=[\widetilde{\sigma} \rho, \widetilde{\sigma} \theta] .
$$

The bracket $[., .]_{\pi}$ satisfies also the Leibnitz rule

$$
[\rho, f \theta]_{\pi}=f[\rho, \theta]_{\pi}+\widetilde{\sigma}(\rho)(f) \theta
$$

and it results that $\left(E^{*},[., .]_{\pi}, \widetilde{\sigma}\right)$ is a Lie algebroid [14].

Next, we can define the contravariant exterior differential $\mathrm{d}^{\pi}: \bigwedge^{k}\left(E^{*}\right) \rightarrow \bigwedge^{k+1}\left(E^{*}\right)$ by

$$
\begin{aligned}
\mathrm{d}^{\pi} \omega\left(s_{1}, \ldots, s_{k+1}\right)= & \sum_{i=1}^{k+1}(-1)^{i+1} \widetilde{\sigma}\left(s_{i}\right) \omega\left(s_{1}, \ldots, \hat{s}_{i}, \ldots, s_{k+1}\right) \\
& +\sum_{1 \leq i<j \leq k+1}(-1)^{i+j} \omega\left(\left[s_{i}, s_{j}\right]_{\pi}, s_{1}, \ldots, \hat{s_{i}}, \ldots, \hat{s}_{j}, \ldots s_{k+1}\right) .
\end{aligned}
$$

In fact, is obtained the cohomology of the Lie algebroid $E^{*}$ with the anchor $\widetilde{\sigma}$ and the bracket $[., .]_{\pi}$ which generalize the Poisson cohomology of Lichnerowicz for Poisson manifolds [9].

\section{The Prolongation of a Lie Algebroid to Its Dual Bundle}

Let $\tau: E^{*} \rightarrow M$ be the dual of $\pi: E \rightarrow M$ and $(E,[\cdot, \cdot], \sigma)$ a Lie algebroid structure over $M$. One can construct a Lie algebroid structure over $E^{*}$, by taking the prolongation of $(E,[\cdot, \cdot], \sigma)$ over $\tau: E^{*} \rightarrow M$ (see [5], [8], [11] and [6]). This structure is given by the following objects:

- The associated vector bundle is $\left(\mathcal{T} E^{*}, \tau_{1}, E^{*}\right)$ where $\mathcal{T} E^{*}=\cup_{u^{*} \in E^{*}} \mathcal{T}_{u^{*}} E^{*}$ with

$\mathcal{T}_{u^{*}} E^{*}=\left\{\left(u_{x}, v_{u^{*}}\right) \in E_{x} \times T_{u^{*}} E^{*} \mid \sigma\left(u_{x}\right)=T_{u^{*}} \tau\left(v_{u^{*}}\right), \tau\left(u^{*}\right)=x \in M\right\}$ and the projection $\tau_{1}: \mathcal{T} E^{*} \rightarrow E^{*}, \tau_{1}\left(u_{x}, v_{u^{*}}\right)=u^{*}$. 
- The Lie algebra structure $[\cdot, \cdot]$ on $\Gamma\left(\mathcal{T} E^{*}\right)$ is defined in the following way: if $\rho_{1}, \rho_{2} \in \Gamma\left(\mathcal{T} E^{*}\right)$ are such that $\rho_{i}\left(u^{*}\right)=\left(X_{i}\left(\tau\left(u^{*}\right)\right), U_{i}\left(u^{*}\right)\right)$ where $X_{i} \in \Gamma(E), U_{i} \in \chi\left(E^{*}\right)$ and $\sigma\left(X_{i}\left(\tau\left(u^{*}\right)\right)=T_{u^{*}} \tau\left(U_{i}\left(u^{*}\right)\right), i=1,2\right.$, then

$$
\left[\rho_{1}, \rho_{2}\right]\left(u^{*}\right)=\left(\left[X_{1}, X_{2}\right]\left(\tau\left(u^{*}\right)\right),\left[U_{1}, U_{2}\right]\left(u^{*}\right)\right) .
$$

- The anchor is the projection $\sigma^{1}: \mathcal{T} E^{*} \rightarrow T E^{*}, \sigma^{1}(u, v)=v$.

Notice that if $\mathcal{T} \tau: \mathcal{T} E^{*} \rightarrow E, \mathcal{T} \tau(u, v)=u$ then $\left(V \mathcal{T} E^{*}, \tau_{\left.1 \mid V \mathcal{T} E^{*}, E^{*}\right)}\right.$ with $V \mathcal{T} E^{*}:=\operatorname{ker} \mathcal{T} \tau$ is a subbundle of $\left(\mathcal{T} E^{*}, \tau_{1}, E^{*}\right)$, called the vertical subbundle. If $\left(x^{i}, \mu_{\alpha}\right)$ are local coordinates on $E^{*}$ at $u^{*}$ and $\left\{s_{\alpha}\right\}$ is a local basis of sections of $\pi: E \rightarrow M$ then a local basis of $\Gamma\left(\mathcal{T} E^{*}\right)$ is $\left\{\mathcal{X}_{\alpha}, \mathcal{P}^{\alpha}\right\}$ where

$$
\mathcal{X}_{\alpha}\left(u^{*}\right)=\left(s_{\alpha}\left(\tau\left(u^{*}\right)\right),\left.\sigma_{\alpha}^{i} \frac{\partial}{\partial x^{i}}\right|_{u^{*}}\right), \quad \mathcal{P}^{\alpha}\left(u^{*}\right)=\left(0,\left.\frac{\partial}{\partial \mu_{\alpha}}\right|_{u^{*}}\right) .
$$

The Lie brackets on the elements of this basis are:

$$
\left[\mathcal{X}_{\alpha}, \mathcal{X}_{\beta}\right]=L_{\alpha \beta}^{\gamma} \mathcal{X}_{\gamma}, \quad\left[\mathcal{X}_{\alpha}, \mathcal{P}^{\alpha}\right]=0, \quad\left[\mathcal{P}^{\alpha}, \mathcal{P}^{\beta}\right]=0
$$

and

$$
\begin{gathered}
\sigma^{1}\left(\mathcal{X}_{\alpha}\right)=\sigma_{\alpha}^{i} \frac{\partial}{\partial x^{i}}, \quad \sigma^{1}\left(\mathcal{P}^{\alpha}\right)=\frac{\partial}{\partial \mu_{\alpha}} \\
\mathrm{d}^{E} x^{i}=\sigma_{\alpha}^{i} \mathcal{X}^{\alpha}, \quad \mathrm{d}^{E} \mu_{\alpha}=\mathcal{P}_{\alpha}, \quad \mathrm{d}^{E} \mathcal{X}^{\gamma}=-\frac{1}{2} L_{\alpha \beta}^{\gamma} \mathcal{X}^{\alpha} \wedge \mathcal{X}^{\beta}, \quad \mathrm{d}^{E} \mathcal{P}_{\alpha}=0
\end{gathered}
$$

where $\left\{\mathcal{X}^{\alpha}, \mathcal{P}_{\alpha}\right\}$ is the dual basis of $\left\{\mathcal{X}_{\alpha}, \mathcal{P}^{\alpha}\right\}$. Also, if $\rho=\rho^{\alpha} \mathcal{X}_{\alpha}+\zeta_{\alpha} \mathcal{P}^{\alpha}$ is a section of $\mathcal{T} E^{*}$, then

$$
\sigma^{1}(\rho)=\sigma_{\alpha}^{i} \rho^{\alpha} \frac{\partial}{\partial x^{i}}+\zeta_{\alpha} \frac{\partial}{\partial \mu_{\alpha}} .
$$

If $u^{*} \in E^{*}$ and $\left(u_{x}, v_{u^{*}}\right) \in E_{x} \times T_{u^{*}} E^{*}$ then

$$
\theta_{E}\left(u^{*}\right)\left(u_{x}, v_{u^{*}}\right)=u^{*}\left(u_{x}\right)
$$

is called the Liouville section. The canonical symplectic section $\omega_{E}$ is defined by

$$
\omega_{E}=-\mathrm{d}^{E} \theta_{E}
$$

and it results that this is a nondegenerate two form and $\mathrm{d}^{E} \omega_{E}=0$.

In the local coordinates it follows that the Liouville section is given by

$$
\theta_{E}=\mu_{\alpha} \mathcal{X}^{\alpha}
$$


and we obtain

$$
\omega_{E}=\mathcal{X}^{\alpha} \wedge \mathcal{P}_{\alpha}+\frac{1}{2} \mu_{\alpha} L_{\beta \gamma}^{\alpha} \mathcal{X}^{\beta} \wedge \mathcal{X}^{\gamma}
$$

We remark that $V \mathcal{T} E^{*}$ is Lagrangian for $\omega_{E}$, i.e., $\omega_{E}\left(\rho_{1}, \rho_{2}\right)=0$, for every vertical sections $\rho_{1}, \rho_{2} \in \Gamma\left(V \mathcal{T} E^{*}\right)$.

Definition 3. The Ehresmann nonlinear connection on $\mathcal{T} E^{*}$ is an almost product structure $\mathcal{N}$ on $\tau_{1}: \mathcal{T} E^{*} \rightarrow E^{*}$ (i.e., a bundle morphism $\mathcal{N}: \mathcal{T} E^{*} \rightarrow \mathcal{T} E^{*}$, such that $\mathcal{N}^{2}=\mathrm{Id}$ ) smooth on $\mathcal{T} E^{*} \backslash\{0\}$ such that $V \mathcal{T} E^{*}=\operatorname{ker}(\operatorname{Id}+\mathcal{N})$.

If $\mathcal{N}$ is a connection on $\mathcal{T} E^{*}$ then $H \mathcal{T} E^{*}=\operatorname{ker}(\operatorname{Id}-\mathcal{N})$ is the horizontal distribution associated to $\mathcal{N}$ and

$$
\mathcal{T} E^{*}=V \mathcal{T} E^{*} \oplus H \mathcal{T} E^{*}
$$

Each $\rho \in \Gamma\left(\mathcal{T} E^{*}\right)$ can be written as $\rho=\rho^{h}+\rho^{v}$ where $\rho^{h}, \rho^{v}$ are sections in the horizontal and respective, vertical subbundles. A connection $\mathcal{N}$ on $\mathcal{T} E^{*}$ induces two projectors $h, v: \mathcal{T} E^{*} \rightarrow \mathcal{T} E^{*}$ such that $h(\rho)=\rho^{h}$ and $v(\rho)=\rho^{v}$ for every $\rho \in \Gamma\left(\mathcal{T} E^{*}\right)$. We have

$$
\begin{gathered}
h=\frac{1}{2}(\operatorname{Id}+\mathcal{N}), \quad v=\frac{1}{2}(\operatorname{Id}-\mathcal{N}) \\
\operatorname{ker} h=\operatorname{im} v=V \mathcal{T} E^{*}, \quad \operatorname{im} h=\operatorname{ker} v=H \mathcal{T} E^{*} \\
h^{2}=h, \quad v^{2}=v, \quad h v=v h=0, \quad h+v=\operatorname{Id} .
\end{gathered}
$$

Locally, a nonlinear connection is expressed as $\mathcal{N}\left(\mathcal{X}_{\alpha}\right)=\mathcal{X}_{\alpha}+2 \mathcal{N}_{\alpha \beta} \mathcal{P}^{\beta}$ and $\mathcal{N}\left(\mathcal{P}^{\alpha}\right)=-\mathcal{P}^{\alpha}$, where $\mathcal{N}_{\alpha \beta}=\mathcal{N}_{\alpha \beta}(x, \mu)$ are the local coefficients of $\mathcal{N}$. The local sections $\mathcal{P}^{\alpha}, \alpha=1, \ldots, m$ define a local frame of $V \mathcal{T} E^{*}$, and the sections

$$
\delta_{\alpha}^{*}=\left(\mathcal{X}_{\alpha}\right)^{h}=\mathcal{X}_{\alpha}+\mathcal{N}_{\alpha \beta} \mathcal{P}^{\beta}
$$

generate a local frame of $H \mathcal{T} E^{*}$. The frame $\left\{\delta_{\alpha}^{*}, \mathcal{P}^{\alpha}\right\}$ is a local basis of $\mathcal{T} E^{*}$ called adapted to the direct sum decomposition. The respective dual adapted basis is $\left\{\mathcal{X}^{\alpha}, \delta \mathcal{P}_{\alpha}\right\}$ where

$$
\delta \mathcal{P}_{\alpha}=\mathcal{P}_{\alpha}-\mathcal{N}_{\alpha \beta} \mathcal{X}^{\beta}
$$

Definition 4. A connection $\mathcal{N}$ is called symmetric if $H \mathcal{T} E^{*}$ is Lagrangian for $\omega_{E}$. 
By a straightforward computation, using (6) and (7) we get

$$
\omega_{E}\left(\delta_{\alpha}^{*}, \delta_{\beta}^{*}\right)=\mathcal{N}_{\alpha \beta}-\mathcal{N}_{\beta \alpha}-\mu_{\gamma} L_{\alpha \beta}^{\gamma}
$$

and it result that $\mathcal{N}$ is symmetric if and only if

$$
\mathcal{N}_{\alpha \beta}-\mathcal{N}_{\beta \alpha}=\mu_{\gamma} L_{\alpha \beta}^{\gamma}
$$

Proposition 5. With respect to a symmetric nonlinear connection, the canonical symplectic structure $\omega_{E}$ can be written in the following form

$$
\omega_{E}=\mathcal{X}^{\alpha} \wedge \delta \mathcal{P}_{\alpha}+\mu_{\alpha} L_{\beta \gamma}^{\alpha} \mathcal{X}^{\beta} \wedge \mathcal{X}^{\gamma}
$$

Proof: Using (6) and (8) we get

$$
\omega_{E}=\mathcal{X}^{\alpha} \wedge \delta \mathcal{P}_{\alpha}+\frac{1}{2}\left(\mathcal{N}_{\alpha \beta}-\mathcal{N}_{\beta \alpha}\right) \mathcal{X}^{\alpha} \wedge \mathcal{X}^{\beta}+\frac{1}{2} \mu_{\alpha} L_{\beta \gamma}^{\alpha} \mathcal{X}^{\beta} \wedge \mathcal{X}^{\gamma}
$$

which ends the proof.

Proposition 6. The Lie brackets of the adapted basis $\left\{\delta_{\alpha}^{*}, \mathcal{P}^{\alpha}\right\}$ are

$$
\left[\delta_{\alpha}^{*}, \delta_{\beta}^{*}\right]=L_{\alpha \beta}^{\gamma} \delta_{\gamma}^{*}+\mathcal{R}_{\alpha \beta \gamma} \mathcal{P}^{\gamma}, \quad\left[\delta_{\alpha}^{*}, \mathcal{P}^{\beta}\right]=-\frac{\partial \mathcal{N}_{\alpha \gamma}}{\partial \mu_{\beta}} \mathcal{P}^{\gamma}, \quad\left[\mathcal{P}^{\alpha}, \mathcal{P}^{\beta}\right]=0
$$

where

$$
\mathcal{R}_{\alpha \beta \gamma}=\delta_{\alpha}^{*}\left(\mathcal{N}_{\beta \gamma}\right)-\delta_{\beta}^{*}\left(\mathcal{N}_{\alpha \gamma}\right)-L_{\alpha \beta}^{\varepsilon} \mathcal{N}_{\varepsilon \gamma}
$$

Proof: Using (7) we obtain

$$
\left[\delta_{\alpha}^{*}, \delta_{\beta}^{*}\right]=\left(\sigma_{\alpha}^{i} \frac{\partial \mathcal{N}_{\beta \gamma}}{\partial x^{i}}-\sigma_{\beta}^{i} \frac{\partial \mathcal{N}_{\alpha \gamma}}{\partial x^{i}}+\mathcal{N}_{\alpha \delta} \frac{\partial \mathcal{N}_{\beta \gamma}}{\partial \mu_{\delta}}-\mathcal{N}_{\beta \delta} \frac{\partial \mathcal{N}_{\alpha \gamma}}{\partial \mu_{\delta}}\right) \mathcal{P}^{\gamma}+L_{\alpha \beta}^{\varepsilon} \mathcal{X}_{\varepsilon}
$$

and putting $\mathcal{X}_{\varepsilon}=\delta_{\varepsilon}^{*}-\mathcal{N}_{\varepsilon \gamma} \mathcal{P}^{\gamma}$ we get $\left[\delta_{\alpha}^{*}, \delta_{\beta}^{*}\right]=L_{\alpha \beta}^{\gamma} \delta_{\gamma}^{*}+\mathcal{R}_{\alpha \beta \gamma} \mathcal{P}^{\gamma}$.

The curvature of a connection $\mathcal{N}$ on $\mathcal{T} E^{*}$ is given by $\Omega=-\mathrm{N}_{h}$ where $h$ is horizontal projector and $\mathrm{N}_{h}$ is the Nijenhuis tensor of $h$, given by

$$
\mathrm{N}_{h}(\theta, \rho)=[h \theta, h \rho]-h[h \theta, \rho]-h[\theta, h \rho]+h^{2}[\theta, \rho] .
$$

Remark 7. In the local coordinates we get

$$
\Omega=-\frac{1}{2} \mathcal{R}_{\alpha \beta \gamma} \mathcal{X}^{\alpha} \wedge \mathcal{X}^{\beta} \otimes \mathcal{P}^{\gamma}
$$

where $\mathcal{R}_{\alpha \beta \gamma}$ is given by (10) and is called the curvature tensor of $\mathcal{N}$. 
Proof: Since $h^{2}=h$ we obtain

$$
\Omega\left(h \rho_{1}, h \rho_{2}\right)=-v\left[h \rho_{1}, h \rho_{2}\right], \quad \Omega\left(h \rho_{1}, v \rho_{2}\right)=\Omega\left(v \rho_{1}, v \rho_{2}\right)=0
$$

and in local coordinates we get

$$
\Omega\left(\delta_{\alpha}^{*}, \delta_{\beta}^{*}\right)=-v\left[\delta_{\alpha}^{*}, \delta_{\beta}^{*}\right]=-\mathcal{R}_{\alpha \beta \gamma} \mathcal{P}^{\gamma}
$$

which concludes the proof.

Remark 8. The curvature satisfies the Bianchi identity

$$
\mathcal{R}_{\alpha \beta \gamma}+\mathcal{R}_{\beta \gamma \alpha}+\mathcal{R}_{\gamma \alpha \beta}=0
$$

Proof: By direct computation, using relation (10) and structure equations given by (1).

The curvature is an obstruction to the integrability of $H \mathcal{T} E^{*}$, understanding that a vanishing curvature entails that horizontal sections are closed under the Lie algebroid bracket of $\mathcal{T} E^{*}$. We have

Remark 9. $H \mathcal{T} E^{*}$ is integrable if and only if the curvature vanishes.

The integrability conditions for the almost product structure $\mathcal{N}$ is given by the vanishing of the associated Nijenhuis tensor $\mathrm{N}_{\mathcal{N}}$. By a straightforward computation we obtain

$$
\mathrm{N}_{\mathcal{N}}\left(\mathcal{P}^{\alpha}, \mathcal{P}^{\beta}\right)=0, \quad \mathrm{~N}_{\mathcal{N}}\left(\delta_{\alpha}^{*}, \mathcal{P}^{\beta}\right)=0, \quad \mathrm{~N}_{\mathcal{N}}\left(\delta_{\alpha}^{*}, \delta_{\beta}^{*}\right)=4 \mathcal{R}_{\alpha \beta \gamma} \mathcal{P}^{\gamma}
$$

Thus

$$
\mathrm{N}_{\mathcal{N}}=-2 \mathcal{R}_{\alpha \beta \gamma} \mathcal{X}^{\alpha} \wedge \mathcal{X}^{\beta} \otimes \mathcal{P}^{\gamma}
$$

and it results that the distribution $H \mathcal{T} E^{*}$ is integrable if and only if the almost product structure $\mathcal{N}$ is integrable.

\subsection{Canonical Poisson Structure}

On the Lie algebroid $\left(\mathcal{T} E^{*},[],, \sigma^{1}\right)$ we have the canonical symplectic section $\omega_{E}$ given by (6) which induces a vector bundle isomorphism

$$
\natural_{\omega_{E}}: E^{*} \rightarrow E, \quad i_{\zeta} \omega_{E} \in E^{*} \rightarrow \zeta \in E
$$


Definition 10. The canonical Poisson bivector is given by

$$
\Lambda=\natural_{\omega_{E}} \omega_{E} .
$$

It follows that

$$
\Lambda(\mathrm{d} F, \mathrm{~d} G)=-\omega_{E}(\natural(\mathrm{d} F), \downarrow(\mathrm{d} G)), \quad F, G \in C^{\infty}\left(E^{*}\right)
$$

and in local coordinates we get

$$
\Lambda=\mathcal{P}^{\alpha} \wedge \mathcal{X}_{\alpha}+\frac{1}{2} \mu_{\alpha} L_{\beta \gamma}^{\alpha} \mathcal{P}^{\beta} \wedge \mathcal{P}^{\gamma}
$$

Remark 11. The Schouten-Nijenhuis bracket $[\Lambda, \Lambda]$ leads, locally, to the expression

$$
\frac{1}{3} \sum_{(\alpha, \beta, \gamma)}\left(\sigma_{\alpha}^{i} \frac{\partial L_{\beta \gamma}^{\varepsilon}}{\partial x^{i}}+L_{\alpha \delta}^{\varepsilon} L_{\beta \gamma}^{\delta}\right) \mu_{\varepsilon} \mathcal{P}^{\beta} \wedge \mathcal{P}^{\alpha} \wedge \mathcal{P}^{\gamma}
$$

and $[\Lambda, \Lambda]=0$ follows from the structure equations on the Lie algebroid (1).

Definition 12. Let us consider a Poisson bivector on E given by (2), then the horizontal lift of $W$ to $\mathcal{T} E^{*}$ is the bivector defined by

$$
W^{H}=\frac{1}{2} w^{\alpha \beta}(x) \delta_{\alpha}^{*} \wedge \delta_{\beta}^{*} .
$$

Proposition 13. The horizontal lift $W^{H}$ is a Poisson bivector if and only if $W$ is a Poisson bivector on $E$ and

$$
w^{\alpha \beta} w^{\gamma \delta} \mathcal{R}_{\beta \gamma \varepsilon}=0
$$

Proof: The Poisson condition $[W, W]=0$ leads to the relation

$$
\sum_{(\alpha, \varepsilon, \delta)}\left(w^{\alpha \beta} w^{\gamma \delta} L_{\beta \gamma}^{\varepsilon}+w^{\alpha \beta} \sigma_{\beta}^{i} \frac{\partial w^{\varepsilon \delta}}{\partial x^{i}}\right)=0
$$

and $\left[W^{H}, W^{H}\right]=0$ yields

$$
\sum_{(\varepsilon, \delta, \alpha)}\left(w^{\alpha \beta} w^{\gamma \delta} L_{\beta \gamma}^{\varepsilon}+w^{\alpha \beta} \sigma_{\beta}^{i} \frac{\partial w^{\varepsilon \delta}}{\partial x^{i}}\right) \delta_{\varepsilon}^{*} \wedge \delta_{\alpha}^{*} \wedge \delta_{\delta}^{*}+w^{\alpha \beta} w^{\gamma \delta} \mathcal{R}_{\beta \gamma \varepsilon} \mathcal{P}^{\varepsilon} \wedge \delta_{\alpha}^{*} \wedge \delta_{\gamma}^{*}=0
$$

which ends the proof.

Recall that two Poisson structures are said to be compatible if the bivectors $w_{1}$ and $w_{2}$ satisfy the condition

$$
\left[w_{1}, w_{2}\right]=0
$$


Proposition 14. If $W^{H}$ is a Poisson bivector and $\mathcal{N}$ is a symmetric nonlinear connection, then $W^{H}$ is compatible with the canonical Poisson structure $\Lambda$ if and only if the following relations fulfilled

$$
\begin{gathered}
\sigma_{\gamma}^{i} \frac{\partial \omega^{\alpha \beta}}{\partial x^{i}}+\omega^{\varepsilon \alpha}\left(\frac{\partial N_{\varepsilon \gamma}}{\partial \mu_{\beta}}-L_{\varepsilon \gamma}^{\beta}\right)-\omega^{\varepsilon \beta}\left(\frac{\partial N_{\varepsilon \gamma}}{\partial \mu_{\alpha}}-L_{\varepsilon \gamma}^{\alpha}\right)=0 \\
\omega^{\varepsilon \alpha} \mathcal{R}_{\alpha \gamma \delta}=0 .
\end{gathered}
$$

Proof: If $\mathcal{N}$ is symmetric then $\mathcal{N}_{\alpha \beta}-\mathcal{N}_{\beta \alpha}=\mu_{\gamma} L_{\alpha \beta}^{\gamma}$ and with respect with the basis $\left\{\delta_{\alpha}^{*}, \mathcal{P}^{\alpha}\right\}$ it results

$$
\Lambda=\mathcal{P}^{\alpha} \wedge \delta_{\alpha}^{*}
$$

By a straightforward computation we obtain that the relation $\left[W^{H}, \Lambda\right]=0$ is equivalent with relations (11) and (12).

\section{Acknowledgements}

The author wishes to express his thanks to the referee for many useful remarks concerning this paper. Also, many thanks to I. Mladenov for the hospitality during the $10^{\text {th }}$ International Conference on Geometry, Integrability and Quantization, Varna 2008, where this paper has been communicated.

\section{References}

[1] Fernandes R., Connections in Poisson Geometry I: Holonomy and Invariants, J. Diff. Geom. 54 (2000) 303-365.

[2] Fernandes R., Lie Algebroids, Holonomy and Characteristic Classes, Adv. Math. 170 (2002) 119-179.

[3] Grabowski J. and Urbanski P., Tangent and Cotangent Lift and Graded Lie Algebra Associated with Lie Algebroids, Ann. Global Anal. Geom. 15 (1997) 447-486.

[4] Grabowski J. and Urbanski P., Lie Algebroids and Poisson-Nijenhuis Structures, Rep. Math. Phys. 40 (1997) 195-208.

[5] Higgins P. and Mackenzie K., Algebraic Constructions in the Category of Lie Algebroids, J. Alg. 129 (1990) 194-230.

[6] Hrimiuc D. and Popescu L., Nonlinear Connections on Dual Lie Algebroids, Balkan J. Geom. Appls. 11 (2006) 73-80. 
[7] Kosmann-Schwarzbach Y., Poisson Manifolds, Lie Algebroids, Modular Classes: A Survey, SIGMA 4 (2008) 005.

[8] De Leon M., Marrero J. and Martinez E., Lagrangian Submanifolds and Dynamics on Lie Algebroids, J. Phys. A: Math. Gen. 38 (2005) 241-308.

[9] Lichnerowicz A., Les Variétés de Poisson et Leurs Algèbres de Lie Associées, J. Diff. Geom. 12 (1977) 253-300.

[10] Mackenzie K., Lie Groupoids and Lie Algebroids in Differential Geometry, London Mathematical Society Lecture Note Series, vol. 124, Cambridge, 1987.

[11] Martinez E., Lagrangian Mechanics on Lie Algebroids, Acta Appl. Math. 67 (2001) 295-320.

[12] Mitric G., Poisson Structures on Cotangent Bundle, IJMMS 29 (2003) 18331855.

[13] Vaisman I., Lectures on the Geometry of Poisson Manifolds, Progress in Math. 118, Birkhäuser, Berlin, 1994.

[14] Vaisman I., Complementary 2-Forms of Poisson Structures, Compositio Mathematica 101 (1996) 55-75.

[15] Weinstein A., The Local Structure of Poisson Manifolds, J. Diff. Geom. 18 (1983) 523-557.

Liviu Popescu

Department of Applied Mathematics in Economy

University of Craiova

13, A. I. Cuza st., 200585 Craiova

ROMANIA

E-mail address: liviupopescu@central.ucv.ro 